\title{
Aménager un espace idéalisé : identité et conflits dans la campagne anglaise
}

Planning process in an idealised space: identity and conflicts in the English countryside

\section{Mark Bailoni}

\section{(2) OpenEdition}

Journals

Édition électronique

URL : http://journals.openedition.org/rge/3739

DOI : $10.4000 /$ rge.3739

ISSN : $2108-6478$

Éditeur

Association des géographes de l'Est

Édition imprimée

Date de publication : 31 décembre 2012

ISSN : 0035-3213

\section{Référence électronique}

Mark Bailoni, «Aménager un espace idéalisé : identité et conflits dans la campagne anglaise », Revue Géographique de l'Est [En ligne], vol. 52 / 3-4 | 2012, mis en ligne le 01 juillet 2013, consulté le 08 septembre 2020. URL : http://journals.openedition.org/rge/3739; DOI : https://doi.org/10.4000/rge 3739

Ce document a été généré automatiquement le 8 septembre 2020

Tous droits réservés 


\section{Aménager un espace idéalisé : identité et conflits dans la campagne anglaise}

Planning process in an idealised space: identity and conflicts in the English countryside

Mark Bailoni

\section{Introduction}

1 Des tensions politiques et des contestations parfois virulentes semblent désormais apparaitre systématiquement dès qu'un projet d'aménagement est proposé. Des citoyens, des élus, des entreprises, des écologistes, rassemblés en diverses associations et groupes de pression, se mobilisent pour soutenir ou pour condamner tel ou tel projet. Ainsi, chacun entend défendre ses intérêts, pour justifier la nécessité du projet, ou au contraire pour dénoncer son inutilité, son coût exorbitant, ses nuisances potentielles ou sa dangerosité sur les équilibres environnementaux, voire sur la santé des riverains. Apparu dans les années 1960, ce type de contestation accompagne désormais quasiment tout type de projet d'aménagement, à toutes les échelles (du projet très local au programme national voire international), et dans toutes les régions du monde, y compris dans les Etats du Sud (conflits autour de la construction de barrages au Brésil ou en Inde par exemple). A travers les tensions, les rivalités de pouvoir et les enjeux qu'il suscite, à travers les représentations contradictoires et les perceptions qu'il véhicule, l'aménagement du territoire est devenu une véritable question géopolitique (Subra, 2007).

2 La question du conflit d'aménagement, liée et parfois confondue à celles du conflit d'usage ou du conflit environnemental, est particulièrement étudiée depuis les années 1990, notamment par des géographes et des aménageurs (Charlier, 1999 ; Dziedzicki, 2001 ; Le Caro, 2002 ; Lecourt, 2003 ; Guérin, 2005 ; Subra 2007) ${ }^{1}$. Ces travaux de plus en plus nombreux démontrent la multiplication de ces conflits fondamentalement liés au 
territoire. Ce phénomène est la conséquence de facteurs sociaux, politiques, culturels et identitaires, qui incitent les citoyens à se mobiliser pour défendre de nouvelles préoccupations notamment environnementales, patrimoniales et sanitaires. Il illustre également une forme de "banalisation de la contestation" (Subra, 2007), puisque aucun type d'équipement ou d'aménagement ne semble désormais épargné, même s'il répond aux préoccupations environnementales (éoliennes ou écoquartiers par exemple).

3 Ces « aménagements rejetés " (Subra, 2007) sont parfois dénoncés pour leur impact à un niveau global. Mais ils sont aussi très souvent contestés plus localement pour leurs possibles nuisances (bruits, pollutions), pour leur éventuelle dangerosité, et au-delà, pour des considérations sociales, politiques, patrimoniales, esthétiques ou écologiques. Ce type de contestation locale relève du phénomène NIMBY (Not In My BackYard). Dans le discours politique ou dans celui des acteurs impliqués dans un conflit d'aménagement, l'usage de l'acronyme NIMBY est souvent stigmatisant et péjoratif, dévalorisant une opposition locale jugée trop égoïste, car allant à l'encontre de l'intérêt général. Au-delà de toute interprétation, l'attitude NIMBY apparait souvent comme une réaction spontanée pour défendre un patrimoine, une qualité de vie, le calme d'un territoire, la beauté d'un paysage ou la valeur d'une propriété, potentiellement menacés par un projet d'aménagement. Dans ce type de contestation, les arguments peuvent converger avec des préoccupations environnementales, des interprétations politiques ou des modèles de développement, qui dépassent le simple cadre local.

Si désormais tous les types de territoires sont concernés (Subra, 2007), certains espaces restent particulièrement sensibles à ce phénomène. A travers les multiples travaux de recherche portant sur des aspects généraux ou sur des études de cas, les espaces périurbains apparaissent en effet comme un cadre privilégié pour les conflits d'aménagement (Lecourt, Faburel, 2005 ; Guérin, 2005 ; Torre, Lefranc, 2006 ; Pistre, 2010 ; Von der Dunk et al., 2011 ; Bailoni et al., 2012). Sans revenir sur la notion de périurbanisation difficilement définissable de manière consensuelle et parfois contestée (Tomasin, 2001; Poulot, 2008), le processus proprement dit bouleverse les territoires et les paysages, mais également les mentalités et les perceptions (Spiers, 2009). L'aménagement des territoires périurbains, "tiers-espace» (Vanier, 2001) multifonctionnels, est particulièrement complexe et sensible, tant l'opposition ville/ campagne est parfois conflictuelle. L'extension ou l'influence de la ville peuvent apparaitre comme une contrainte, voire une menace pour les espaces ruraux, impression renforcée quand la ville rejette ses nuisances - déchetterie, prison, rocade autoroutière, aéroport, etc. - sur ses marges (Subra, 2007). Les néo-ruraux ont parfois des valeurs, des attentes et une vision du territoire et du paysage différentes des populations plus anciennement implantées (Gilbert, 2010). Ces perceptions du territoire - élément fondamental des représentations identitaires - parfois antagonistes peuvent être sources de tensions. Cependant, une fois installés à la campagne, les néo-ruraux apparaissent souvent comme de farouches défenseurs des paysages ruraux, s'impliquant volontiers dans les mobilisations NIMBY et la lutte contre l'étalement urbain (Hall, al., 1973; Pistre, 2010). Ce comportement n'est pas paradoxal: il peut s'interpréter comme la volonté de défendre l'idéal et le cadre de vie que ces néo-ruraux sont venus trouver et qu'ils ne sont pas prêts à sacrifier. Ce phénomène renforce la particularité des conflits d'aménagement dans les espaces périurbains. 
5 Si ces phénomènes conflictuels existent dans la frange périurbaine de très nombreuses villes européennes, moyennes ou grandes, l'aménagement de certains territoires se révèle être un enjeu géopolitique majeur. C'est le cas du Sud-Est anglais, l'une des régions les plus riches et les plus dynamiques d'Europe. Autour de Londres, ville mondiale saturée, se confrontent enjeux nationaux, régionaux et locaux, intérêts privés et communs, acteurs publics, économiques, politiques, associatifs, écologistes et citoyens. Dans ce territoire où ont été expérimentées de nombreuses innovations de l'aménagement périurbain dès la première moitié $\mathrm{du}$ XXe siècle, la question est d'autant plus sensible que le monde rural évoque chez les Anglais des représentations extrêmement positives, presque mythifiées. "Vivre à la campagne » apparait comme un idéal pour la majorité d'entre eux, qui entretiennent avec leur countryside une véritable " histoire d'amour» (Champion, 2001). La pression périurbaine est ainsi très forte, tout comme les mobilisations pour tenter de protéger ces territoires idéalisés.

Ainsi, des contestations NIMBY apparaissent dès qu'un projet d'aménagement est proposé dans la frange périurbaine de Londres. D'ailleurs, l'expression « NIMBY » aurait été médiatisée grâce à Nicholas Ridley, le secrétaire d'Etat à l'environnement de Margaret Thatcher, qui l'a beaucoup utilisée à la fin des années 1980, pour dénoncer l'attitude des opposants à la construction de nouveaux logements autour de Londres et au grignotage des espaces ruraux. Critiquant cette attitude, il expliquait que les paysages ruraux anglais étaient essentiellement le résultat du travail humain. Selon lui, « faire de la campagne un musée reviendrait à nier son passé » (N. Ridley, 1988, cité par $B B C$ News, 2002). Mais l'histoire est parfois facétieuse puisque quelques années plus tard, il s'est personnellement mobilisé pour dénoncer la construction de nouvelles maisons face à sa résidence secondaire dans les Cotswolds. Avec la généralisation des mobilisations NIMBY souvent passionnées et très populaires, la campagne anglaise peut alors apparaitre comme une forme de sanctuaire difficilement aménageable et transformable.

7 Cet article entend analyser ces conflits d'aménagement et le phénomène NIMBY dans les espaces ruraux périphériques de Londres, en étudiant leurs particularités et leurs enjeux, dans ce contexte d'idéalisation, voire de sacralisation de la campagne considérée comme un véritable symbole de l'identité anglaise.

\section{Le mythe de la campagne anglaise}

8 Dans l'abondante littérature sur la définition de l'identité ou de la nation anglaise ${ }^{2}$, les paysages ruraux et les références à la campagne sont très souvent cités comme des repères incontournables. Ils auraient notamment contribué à forger "l'esprit anglais ", à la manière des Highlands pour les Ecossais, constituant ainsi à un véritable patrimoine national (Bailoni, 2007). Ainsi, selon Patrick Abercrombie, «le plus grand monument historique en notre possession, la chose la plus essentielle qui constitue l'Angleterre, est la campagne » (citation de 1926 reprise par Salomon Carvin, 2006). Cette représentation reste actuelle, puisque le livre blanc du gouvernement Major " Rural England: A Nation committed to a living countryside» (1995) commençait ainsi : «la campagne constitue un caractère immuable de l'Angleterre » (cité par Howkins, 2001). Ce document définissait la campagne comme une "ressource nationale", non pas en termes économiques, mais d'un point de vue culturel, identitaire et patrimonial. Ainsi dans le discours NIMBY contemporain et dans la contestation de tel ou tel projet 
d'aménagement dans l'espace périurbain de Londres, la préservation des paysages et de l'image même de la campagne anglaise apparait comme un argument fondamental, particulièrement mobilisateur et populaire. Mais qu'est-ce que la campagne anglaise? Quelles sont ses particularités qui la distingueraient d'autres paysages ruraux?

\section{A. Un territoire idéalisé}

Telle qu'elle est évoquée dans ce type de discours, la campagne anglaise - l'idée d'une campagne britannique n'apparait jamais - est pittoresque, verte, bocagée, jardinée et parsemée de pubs à la cheminée fumante et de cottages aux toits de chaume. C'est une campagne harmonieuse, préservée de la modernité, qui ressemble beaucoup à un tableau de Constable ou d'un autre peintre paysagiste ${ }^{3}$ du XIXe, ou à une description tirée d'un roman de Jane Austen (figure 1). Cette représentation se retrouve même lors de la cérémonie d'ouverture des JO de Londres en 2012, dans l'évocation de l'Angleterre d'avant l'industrialisation et l'urbanisation. Cette représentation s'apparente donc à un cliché. Même si ces paysages rêvés ne correspondent pas à la pluralité des paysages de l'Angleterre et au cadre de vie de la très grande majorité des Anglais, ils entrainent une certaine nostalgie et certaines représentations qui pourraient s'apparenter à des sentiments identitaires.

Figure 1 : Représentations clichés de la campagne anglaise, des paysages idéalisés

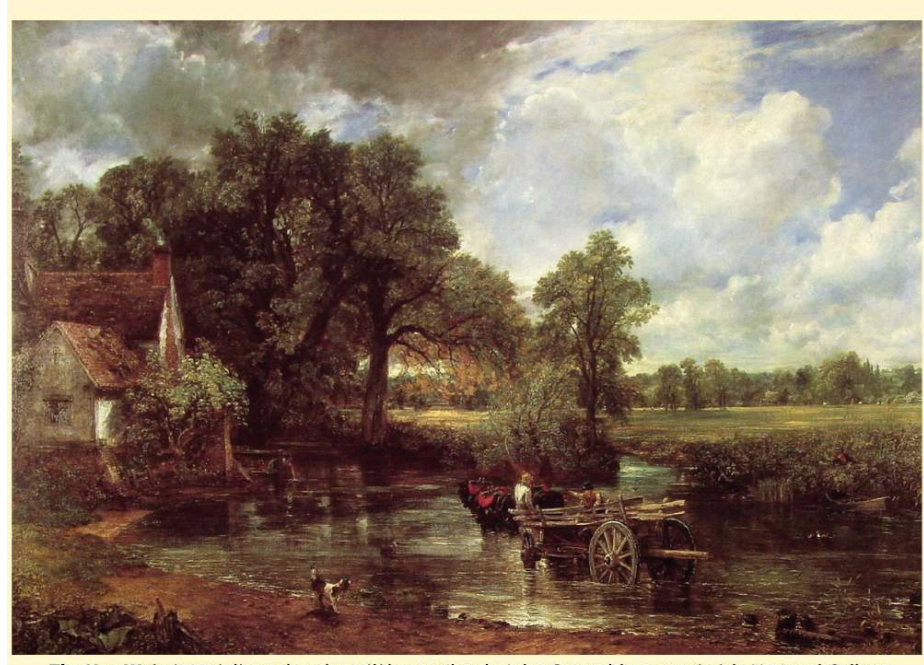

The Hay Wain (1821), I'une des plus célèbres toiles de John Constable, exposée à la National Gallery de Londres et représentant un paysage rural au bord de la Stour, aux confins de l'Essex et du Suffolk

" Dans un site magnifique et bien abrité, se trouvait la ferme d'Abbey Mill devant laquelle s'étendaient de belles prairies où la rivière courait en larges boucles.

Le spectacle était vraiment charmant, doux à l'oeil et doux à l'esprit. On avait ici une vision parfaite de la verdure anglaise, des cultures anglaises et du confort anglais, tout cela agrémenté d'un soleil étincelant sans en être accablant. »

Extrait de Emma (1815), de Jane Austen (cité dans Mathis, 2010, p.52). L'auteur se serait inspirée des paysages du Surrey.

Certaines valeurs sociales renforcent cette vision très positive de la campagne. Selon J. Murdoch (1997), habiter à la campagne apparait comme un idéal : c'est vivre à la fois dans un "environnement naturel » et dans "une véritable communauté ». Au monde rural est associée la représentation d'une société plus solidaire, où le partage, l'entraide et le travail commun sont des valeurs plus importantes que la réussite individuelle, et 
transcendent les catégories sociales. Cette interprétation idéalisée des valeurs rurales s'oppose à une vision particulièrement négative de la ville, perçue comme un cadre de vie difficile, laid et déshumanisé (Bunce, 1994). Cette représentation de deux mondes antagonistes apparait dès le XVIIIe siècle, dès le début de l'urbanisation (Mathis, 2010), et se renouvelle depuis. Les difficultés sociales et les incivilités contemporaines succèdent aux descriptions de Dickens, les immeubles de verre froids et standardisés d'aujourd'hui remplacent les cheminées et les rues sombres des vieux quartiers industriels. Au-delà d'un patrimoine ou d'un héritage à défendre, ces paysages ruraux, tels qu'ils sont imaginés et revendiqués, incarnent aussi un cadre social idyllique. Par cette idéalisation, la campagne anglaise est donc véritablement mythifiée.

11 La ruralité et la "nature " sont indissociables de "l'anglicité » (Englishness) (Matless, 1998; Hawkins, 2001), au moins depuis le XVIIIe siècle (Mathis, 2010). Cela pourrait apparaître comme un paradoxe dans le pays de la Révolution Industrielle, où l'agriculture est depuis longtemps très marginale dans l'économie nationale. En effet, si officiellement 18,9\% des Anglais sont ruraux (en 2010) ${ }^{4}$, le secteur agricole emploie moins de $1 \%$ de la population active et ne représente que $0,7 \%$ des revenus du pays. Toutefois, comme le souligne Alun Howkins (2007), les revenus générés par les séjours d'une journée - c'est-à-dire sans nuitée - dans les espaces ruraux anglais représentent le double de ceux de l'agriculture. Aller à la campagne pour faire de la randonnée et du cyclisme - activités accessibles aux classes moyennes et populaires - ou pour chasser s'apparente à une forme de culture populaire, au cœur de l'identité anglaise $\mathrm{e}^{5}$. La remise en cause de certaines traditions, qui pourraient apparaître obsolètes, est alors loin d'être évidente. La manifestation réunissant 400.000 personnes en 2002 contre la politique rurale du gouvernement - sous-entendu contre le projet de règlementer sévèrement la chasse à courre - en est un bon exemple (BBC News, 22 septembre 2002). L'économie du tourisme et des loisirs est donc considérable dans les espaces ruraux. La campagne anglaise tend ainsi à incarner davantage un espace de loisirs et de détente, qu'un espace de production agricole (Howkins, 2007). La préservation de la campagne et de ses paysages est donc une préoccupation qui dépasse les seules populations rurales. De nombreux urbains, qui fréquentent ces espaces pour leurs loisirs, sont également sensibilisés. Les représentations identitaires liées à ces paysages rêvés et à leurs usages expliquent aussi sans doute la popularité de certains mouvements de protection des espaces ruraux, et la virulence de certains conflits d'aménagements et de certaines contestations NIMBY.

\section{B. La défense des espaces ruraux : entre conscience environnementale, protection du patrimoine et intérêts locaux}

Dans sa remarquable étude, Charles-François Mathis (2010) montre le lien entre l'idéalisation de ces paysages d'une campagne idyllique, devenus un symbole essentiel de l'identité nationale anglaise, et l'émergence d'une conscience environnementale dès le XIXe siècle. Le Royaume-Uni devient précurseur pour la protection de la nature, avec l'apparition des premières associations à l'époque victorienne. Le National Trust for Places of Historic Interest or Natural Beauty - plus couramment appelé National Trust - est ainsi créé en 1894, par Octavia Hill, militante pour l'amélioration des conditions de vie des citadins et surtout pour la préservation d'espaces naturels accessibles autour des grandes villes. Fondation indépendante, il rachète et protège des sites historiques et naturels pour les ouvrir au public. Il possède aujourd'hui plus de 350 sites, 
essentiellement localisés en milieu rural. Le National Trust administre ainsi 255.000 hectares d'espaces naturels et de campagne, remarquables d'un point de vue environnemental, paysagé ou historique, et 1.141 kilomètres de littoraux. C'est un acteur incontournable et très influent de la protection du patrimoine et des paysages en Angleterre. En effet, le National Trust compte 3,8 millions de membres en 2011 - son record - et rassemble plus de 61.000 bénévoles. Cette popularité sans cesse réaffirmée montre l'intérêt des Anglais pour leur patrimoine, notamment rural.

Pour protéger les espaces naturels et ruraux, des associations spécifiques et des groupes de pression se sont également constitués. La Campaign to Protect Rural England (CPRE) est probablement le plus important et le plus influent sur les politiques d'aménagement périurbain en Angleterre. Sa création par Patrick Abercrombie en 1926 est liée aux premières propositions pour protéger les espaces ruraux de l'étalement urbain (Matless, 1998). Très impliquée dans les politiques d'aménagement de l'aprèsguerre, la CPRE reste très active, évoluant vers de nouvelles préoccupations (protection des espèces, réduction des nuisances sonores et visuelles et de la pollution lumineuse, impacts de la PAC, pratiques agricoles, développement durable, etc.). Structurée sur un réseau d'antennes régionales et locales, la CPRE s'implique également dans de nombreux conflits d'aménagement, soutenant parfois des mouvements nimbystes, pour contester des projets qui, selon elle, menacent la beauté et la tranquillité des espaces ruraux. D'ailleurs, reflet du phénomène NIMBY, la majorité des membres de la CPRE sont, depuis les années 1960, des néo-ruraux (Salomon Carvin, 2006). Ses détracteurs critiquent son implication dans ce type de contestation souvent jugée égoïste, mais aussi plus généralement sa vision de la campagne anglaise parfois décrite comme désuète, arriérée et loin des réalités contemporaines. Toutefois, l'organisation dément toute ligne traditionaliste ou conservatrice (Spiers, 2009). Pour se détacher d'une image trop exclusive et trop fermée, la CPRE entend également s'intéresser à l'aménagement urbain, en prônant notamment une ville plus dense et plus harmonieuse, améliorant la qualité de vie de ses habitants. Mieux aménager la ville est en effet la meilleure stratégie pour protéger les espaces ruraux et freiner la périurbanisation (Salomon Carvin, 2006). Malgré certaines critiques, la CPRE reste très écoutée par les aménageurs, les décideurs politiques et économiques de tous bords. Elle s'est imposée comme une référence, voire comme une autorité incontournable, pour toute politique concernant l'espace rural. David Matless (1998) décrit son action comme « un travail d'expertise publique de référence pour façonner un paysage simultanément moderne et traditionnel ».

D'autres groupes de pression ont été créés pour défendre un certain mode de vie rural. Ainsi, la Countryside Alliance a été fondée en 1997, pour répondre à l'arrivée au pouvoir du gouvernement Blair, dont le programme prévoyait l'interdiction de la chasse à courre. Elle se présente comme une organisation défendant la vie à la campagne, la pratique d'activités traditionnelles, les agriculteurs ou le maintien d'activités et de services en milieu rural. Mais elle incarne avant tout un groupe de pression défendant la pratique de la chasse et les revendications des chasseurs. Comme la CPRE, la Countryside Alliance conteste toute affiliation politique, puisque que son comité de direction se compose de parlementaires travaillistes et conservateurs. Sa directrice exécutive, la députée Kate Hoey, est d'ailleurs élue dans la circonscription de Vauxhall, située en plein cœur de Londres! Cela montre aussi que les urbains sont mobilisés pour la protection des espaces ruraux. 
15 La Countryside Alliance et la CPRE sont les associations les plus importantes et les plus médiatisées d'un très important réseau de groupes de pression plus ou moins spécialisés et localisés, agissant pour la défense des territoires ruraux, de leurs paysages et des intérêts de leurs populations. Parce qu'ils défendent des valeurs populaires au cœur des représentations identitaires de beaucoup d'Anglais, leur influence politique, notamment sur les questions d'aménagement, est considérable. Depuis l'après-guerre, les gouvernements successifs ont d'ailleurs créé et maintenu des règles spécifiques pour l'aménagement des territoires périurbains.

\section{Comment contrôler et maitriser la périurbanisation ?}

16 Les premières propositions concrètes pour contrôler l'étalement urbain et la croissance de Londres apparaissent dans les années 1920, alors que plusieurs générations d'urbanistes innovants, comme Patrick Geddes, Ebenezer Howard, Thomas Adams, Raymond Unwin, Thomas Sharp ou Clough Williams-Ellis, avaient déjà, d'une manière ou d'une autre, souligné l'importance d'un aménagement raisonné plus en lien avec la nature (Hall, 2002). Le projet le plus novateur, imaginé par plusieurs urbanistes, est de créer une ceinture verte (green belt) autour de Londres, pour contrôler l'expansion de la capitale et pour protéger sa périphérie rurale d'une urbanisation trop rapide et anarchique. Cette idée est mentionnée officiellement en 1935 dans le rapport du Greater London Regional Planning Committee, commission chargée de trouver des solutions pour un aménagement harmonieux du grand Sud-Est. Patrick Abercrombie développe ce projet, notamment dans ses propositions du Greater London Plan de 1944. Mais les premières mesures effectives ne sont prises par le gouvernement qu'à la fin des années 1940, après la parenthèse de la Seconde Guerre mondiale. Ainsi, les premières villes nouvelles sont crées par le New Towns Act de 1946 et la Metropolitan Green Belt est établie autour de Londres, par le Town and Country Planning Act de 1947 (ODPM, 1995). Par ces programmes d'envergure nationale, l'Etat veut aussi encourager la relance économique dans le Nord de l'Angleterre, déjà en difficulté, en freinant la croissance du Sud-Est (Cullingworth, Nadin, 2006).

17 Ainsi, le Royaume-Uni devient précurseur dans la protection des périphéries rurales, faisant du Sud-Est anglais un laboratoire de l'aménagement périurbain (Hall, 2002). A partir de 1955, le modèle de la green belt est reproduit autour d'autres autres grandes conurbations - comme le Grand Manchester, les West Midlands (Birmingham), la Merseyside (Liverpool), etc. - et même de villes moyennes (Gloucester, Burton-uponTrent, etc.). Le périmètre cumulé de ces 14 green belts représente en 2012 plus de 1,6 millions d'hectares, soient environ $13 \%$ de la superficie totale de l'Angleterre, dont 515.000 hectares pour la seule ceinture verte de Londres (DCLG, 2012). En parallèle, l'Etat a aussi construit d'autres villes nouvelles, situées au-delà des ceintures vertes pour former de nouveaux pôles absorbant la croissance urbaine. Des stratégies de contrôle de la périurbanisation sont également imaginées au niveau local. Par exemple, les autorités locales de Cambridge ont mis en place des règles d'urbanisation relativement strictes depuis les années 1950 (Baker, 2010), bien avant l'émergence des pratiques d'un aménagement dit durable.

18 Toutefois, l'efficacité de ces mesures reste discutée. Créer une ceinture verte autour de Londres a certes permis de contrôler et de réguler les projets de construction, mais sans pour autant geler le processus de périurbanisation. Les navetteurs peuvent en 
effet franchir la green belt grâce à l'automobile. Des économistes et des géographes ont même montré que la politique d'endiguement a provoqué certains effets environnementaux, sociaux et économiques particulièrement négatifs (Hall et al., 1973 ; Gilg, 2005 ; Barker, 2006). J. Murdoch et P. Lowe (2003) évoquent un «paradoxe de la protection »: la préservation des espaces ruraux les a rendus encore plus attractifs pour de nombreux urbains décidés à quitter la ville. Conséquence d'une forte demande de logements dans une zone où il est très difficile d'en construire, l'inflation des prix du foncier a été également constatée dès les années 1970 dans le périmètre protégé (Hall et al., 1973). Le statut de ceinture verte aurait ainsi encouragé une forme de périurbanisation réservée aux catégories les plus aisées de la population (Champion, 2001). Le processus de périurbanisation s'est ainsi accompagné d'une forme de gentrification des territoires ruraux, autour de Londres et des autres grandes villes, ou dans des espaces naturels remarquables (Phillips, 1993). La périurbanisation s'apparente alors à " une colonisation socialement sélective » (Richard, 2009), puisque seuls ceux qui en ont les moyens peuvent accéder à "leur préférence résidentielle » (Champion, Fisher, 2004). D'ailleurs, les différents indices statistiques montrent que les espaces périurbains autour de Londres, composant les "Home counties ${ }^{6}$, sont des territoires particulièrement favorisés, sans doute les plus prospères du pays (Bailoni, 2010). Cette pression sur les prix du terrain a ainsi créé des clivages sociaux entre les espaces urbains et périurbains, entre les zones protégées et non-protégées autour de Londres. Ces discontinuités sociales et ce phénomène de gentrification rurale entrainent des ressentiments, et attisent ainsi les conflits d'aménagement dans les franges périurbaines de Londres.

Dans son rapport sur la situation économique du Royaume-Uni, l'OCDE (2011) critique les normes d'aménagement trop strictes imposées par la préservation des green belts. Elles empêcheraient la construction de logements pourtant indispensables, ce qui nuirait au développement économique du pays. L'OCDE préconise des plans d'occupation du sol à une échelle plus fine, préservant l'environnement et les paysages, là où il existe un réel besoin. Certains représentants économiques, notamment des organisations patronales, partagent ce point de vue et réclament une révision des règles d'aménagement autour des grandes villes britanniques (Barclay, 2012). La Home Builders Federation, représentant les intérêts des entrepreneurs du bâtiment, demande quant à elle une profonde révision des limites des zones protégées autour des villes. Au contraire, les associations de protection de l'environnement ou des paysages, comme la CPRE, défendent farouchement les green belts, soulignant leurs réussites, comme la limitation de la consommation d'espaces agricoles et la protection du patrimoine naturel ou historique. Elles estiment que ces ceintures vertes sont essentielles dans la lutte contre le changement climatique (CPRE, Natural England, 2010).

Ainsi, plus de 60 ans après sa mise en place, la préservation de la green belt continue de faire débat au Royaume-Uni. Malgré les pressions économiques et le besoin de logements, les gouvernements successifs ne sont jamais revenus sur son principe, étendant même les périmètres protégés. Dans le National Planning Policy Framework ${ }^{7}$ (DCLG, 2012), document fixant les grandes orientations de l'aménagement au RoyaumeUni, le gouvernement actuel définit d'ailleurs la préservation des green belts comme une enjeu prioritaire : en parallèle au développement économique, il est en effet rappelé la nécessité de défendre et de promouvoir le patrimoine naturel et historique notamment les paysages ruraux -, de favoriser le dynamisme des bourgs ruraux, de concentrer la construction de nouvelles infrastructures ou de nouveaux logements sur 
des friches urbaines, industrielles ou militaires, et d'encourager le développement de cités-jardins. Finalement, les objectifs de cet aménagement, qui est décrit comme "durable », reprennent les grandes idées des précurseurs de la fin du XIXe et du début $\mathrm{du}$ XXe siècle. Programme innovant pour contrôler l'expansion urbaine après-guerre, la politique de la green belt demeure ainsi un principe fondamental de l'aménagement du territoire au Royaume-Uni. Au-delà de ses objectifs premiers, la Metropolitan Green Belt est même devenue un symbole. Ainsi, chaque projet d'aménagement qui empiète potentiellement sur son périmètre provoque de nombreuses réactions dans les territoires concernés, et même au-delà, par la mobilisation d'associations écologistes ou de défense du patrimoine. Dans la frange périurbaine de Londres, les conflits d'aménagement sont ainsi très nombreux, nourri par des contestations NIMBY.

\section{Entre contestations et atermoiements politiques, comment aménager?}

\section{A. La généralisation de la contestation NIMBY}

21 Le développement de la contestation NIMBY, associé à la défense de la campagne anglaise, véritable patrimoine national, complique très fortement les programmes d'aménagement pourtant indispensables dans le grand Sud-Est anglais. L'un des projets les plus dénoncés au cours de ces dernières années est l'extension de l'aéroport d'Heathrow à la périphérie Ouest du Grand Londres. Le mouvement de contestation, très médiatisé, s'opposait au programme de construction de nouvelles infrastructures et surtout d'une troisième piste qui mordraient immanquablement sur des espaces non-bâtis et en partie inclus dans le périmètre de la Green Belt. L'augmentation programmée du trafic provoquerait également davantage de nuisances pour les riverains. Autour de Londres, comme dans d'autres villes, les projets d'aéroport sont sans doute les plus contestés et les plus mobilisateurs, tant le transport aérien est facteur de nuisance et de pollution ${ }^{8}$. Mais, signe de la généralisation du phénomène NIMBY, d'autres types de projets, même respectueux de l'environnement, sont également fortement dénoncés autour de Londres.

Ainsi, chaque projet de construction de logements, quelle qu'en soit la nature, semble systématiquement contesté. Londres souffre pourtant d'un manque criant de logement, qui place le prix des loyers et du foncier à un niveau très élevé, souvent inabordable pour les classes moyennes (Appert et al., 2012). Pour répondre à cette pénurie, les gouvernements Blair et Brown ont lancé plusieurs grands programmes, comme le Thames Gateway et les Growth Areas, prévoyant la création de nouveaux quartiers mêlant logements et activités, dans le grand Sud-Est britannique (Bailoni, 2009). Mais ces projets, symboles de la périurbanisation, ont été immédiatement contestés dans les territoires concernés, où le gouvernement a été accusé de vouloir grignoter des espaces ruraux, de construire dans des périmètres protégés, de défigurer les paysages ou de porter atteinte aux fragiles équilibres environnementaux. Dans ces discours, la défense de la Green Belt et plus généralement de la campagne anglaise est apparue comme un enjeu fondamental et très mobilisateur. Le périmètre de la ceinture verte est d'ailleurs représenté sur les cartes de la figure 2, publiées en 2007 par le quotidien conservateur The Daily Telegraph pour dénoncer les programmes du gouvernement travailliste. 
Figure 2 : La campagne anglaise « menacée » par la périurbanisation

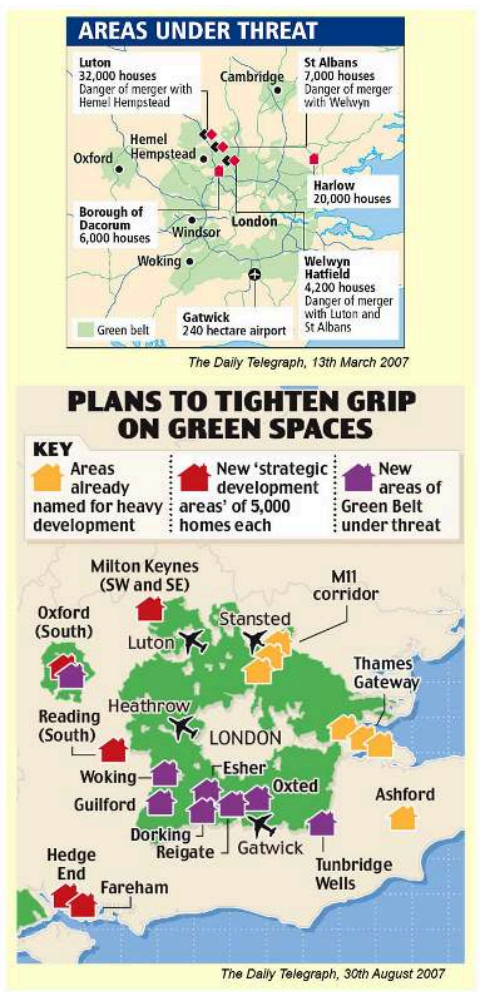

Toutefois, même quand les projets répondent aux principes d'un aménagement dit durable, les réactions d'opposition sont particulièrement vigoureuses. Ainsi, quand le gouvernement travailliste annonce en 2008 la création d'une nouvelle génération de villes-nouvelles, les eco-towns, la contestation se généralise sur les 15 sites envisagés. Pourtant, le projet est particulièrement innovant et respectueux de l'environnement, du bien-être et du cadre de vie des résidents, ressuscitant les principes des citésjardins. En effet, ces villes doivent rester petites (de l'ordre de 10.000 habitants chacune), constituées de bâtiments répondant à des normes strictes de performances énergétiques. Elles doivent mêler résidences, emplois et loisirs, et bénéficier d'un système de transports coordonnés. Enfin, exigence fondamentale de l'urbanisme durable, elles ne doivent pas " consommer de l'espace rural ", puisqu'elles sont toutes programmées sur des friches industrielles ou militaires, situées en dehors de zones classées (Appert et al., 2012). De même, l'aménagement d'un grand réservoir d'eau à proximité d'Oxford fait l'objet d'une très vive contestation locale. Présenté comme nécessaire pour l'approvisionnement de Londres - qui contrairement aux clichés sur le climat anglais manque d'eau - et sans nuisance apparente, ce projet de lac artificiel est pourtant fortement dénoncé (Appert et al., 2012). L'image d'une campagne défigurée pour les besoins de la ville est très utilisée dans le discours des opposants. Ainsi, quel que soit le type de projet proposé, quel que soit le site concerné - qu'il soit dans la Green Belt ou non, classé ou non - la contestation semble systématique, usant d'arguments souvent très proches et mobilisant les mêmes grands groupes de pression (la CPRE, Greenpeace, etc.) en soutien des associations locales.

Beaucoup de projets d'aménagement ont été profondément modifiés, suspendus, voire annulés, au cours de ces dernières années. La contestation locale a sans doute eu une influence certaine sur les élus. Mais le changement du contexte économique et la 
réduction des financements privés et publics, la mobilisation de certains fonds pour financer des Jeux Olympiques qui se sont avérés très couteux, ou le changement de majorité politique dans le pays ont aussi eu des conséquences majeures sur certains projets, notamment les plus contestés. C'est le cas de plusieurs programmes de constructions de nouveaux quartiers, dont la plupart des eco-towns, mais surtout du très médiatique projet d'extension d'Heathrow.

Ce projet était soutenu par la précédente majorité travailliste, mais le gouvernement Cameron l'a suspendu quelques semaines après son arrivée au pouvoir en 2010. C'était l'une des promesses de campagne du parti conservateur. En effet, les territoires concernés - et donc les plus mobilisés contre l'extension d'Heathrow - votent très majoritairement tory, et beaucoup de députés de la majorité sont élus dans les Home counties. De nombreux élus londoniens sont également opposés au projet, en premier lieu le maire Boris Johnson, également conservateur. Si Londres a besoin d'une capacité aéroportuaire majeure pour son rayonnement international, le maire soutient un projet pharaonique : construire un tout nouvel aéroport sur une île artificielle dans l'estuaire de la Tamise. Pour répondre aux lobbys du transport aérien, le gouvernement explore d'autres idées, comme construire de nouvelles pistes à Gatwick ou Stansted (The Guardian, 25 mars 2012), voire transformer le petit aéroport de Lydd sur le littoral du Kent en véritable hub international (The Observer, 26 février 2012). Mais le projet du "Boris airport » comme les autres idées évoquées suscitent tous une forte contestation locale et des réactions de type NIMBY. Pour des questions de rentabilité, des rumeurs circulent même durant l'été 2012 sur un possible revirement du gouvernement Cameron qui soutiendrait finalement la solution d'une troisième piste à Heathrow (The Telegraph, 13 juin 2012).

26 A travers cet exemple, les hésitations et les revirements, suscités par la pression des lobbys économiques, par des considérations politiques et par la mobilisation citoyenne, illustrent les difficultés de l'aménagement dans des territoires périurbains, soucieux de conserver leur qualité de vie et refusant les nuisances, avérées ou non, de la ville. Pour analyser plus précisément ce type de contestation, les discours des acteurs impliqués et les mécanismes de ces conflits, prenons l'exemple du projet de la ligne à grande vitesse HS2.

\section{B. Le conflit de la ligne HS2 : entre intérêt général, contestation NIMBY et considérations politiques}

\section{Un projet présenté comme indispensable}

27 Le réseau ferroviaire à grande vitesse britannique est très limité, puisque la seule ligne existante est celle de l'Eurostar, entre Londres et le tunnel sous la Manche, appelée HS1 (High Speed 1). Les temps de trajet sont ainsi relativement longs entre les principales villes britanniques, alors que le réseau est ancien, datant pour l'essentiel de l'époque victorienne. Avant l'inauguration de la HS1 en 2007, la dernière ligne importante construite dans le pays datait de 1899 (DfT, 2012). De plus, les principaux axes de transport britanniques sont surchargés, voire saturés sur certaines portions. C'est le cas des principales autoroutes ou de certaines lignes ferroviaires, comme la West Coast Main Line, reliant Londres, Birmingham, Liverpool, Manchester et Glasgow. Certes, des opérations de modernisation ont permis d'améliorer certaines dessertes, mais le Royaume-Uni est très en retard par rapport à ses grands voisins européens, paradoxe 
pour le pays pionnier dans d'histoire ferroviaire. Plusieurs projets ont certes émergé, dès les années 1970, mais les choix économiques du pays, la privatisation de British Rail dans les années 1980, le sous-investissement et la crise du secteur, puis sa renationalisation partielle dans les années 2000 n'ont pas permis l'émergence d'une politique de développement ferroviaire à long terme, comme celle du TGV en France.

Pour rattraper ce retard, moderniser le réseau et surtout réduire les encombrements, il faut construire de nouvelles infrastructures. Avec un réseau à grande vitesse, les pouvoirs publics veulent réduire le trafic sur les lignes classiques, et espèrent drainer une partie du transport routier et aérien vers le ferroviaire, grâce à des temps de parcours plus attractifs. Dans le discours officiel, la grande vitesse ferroviaire permettrait aussi de réduire les émissions de carbone, aidant le pays à atteindre ses objectifs environnementaux, et elle favoriserait la réduction les disparités économiques et sociales entre les régions, atténuant le fameux clivage Nord/Sud (DfT, 2012).

La construction d'une seconde ligne à grande vitesse, la HS2 (High Speed 2) est ainsi programmée pour rejoindre Londres et Birmingham. La HS2 sera également connectée avec la ligne Eurostar à travers Londres ${ }^{9}$. Cette première phase devrait aboutir en 2026. Dans un second temps, à l'horizon 2032-33, cette ligne sera prolongée par deux branches, l'une vers Leeds et l'autre vers Manchester. Les premières annonces officielles sur le tracé de ces extensions sont faites en 2013, alors que les consultations et le débat public sont annoncés pour 2014. Par la suite, le réseau pourrait encore être étendu vers le Nord de l'Angleterre et l'Ecosse. Les trains rouleront jusqu'à $400 \mathrm{~km} / \mathrm{h}$ en service. Birmingham sera ainsi à 45 minutes de Londres, contre $1 \mathrm{~h} 24$ actuellement, Manchester à $1 \mathrm{~h} 08$, contre $2 \mathrm{~h} 08$, et Leeds à $1 \mathrm{~h} 28$, contre $2 \mathrm{~h} 20$. Les trajets vers l'Ecosse seront aussi réduits de quasiment une heure. Des trains internationaux pourraient également être mis en service pour relier directement Birmingham et Paris en moins de $3 \mathrm{~h}$ (DfT, 2012).

30 Si aujourd'hui le gouvernement Cameron conduit le projet, il n'en est pas l'instigateur. Les premiers projets concrets d'un prolongement de la HS1 vers le Nord sont apparus au début des années 2000. Après une série de rapports d'expertises, le gouvernement Brown reconnait officiellement la nécessité et la viabilité de construire cette nouvelle ligne en janvier 2009 (DfT, 2009). Il crée alors HS2 Limited, entreprise dépendant entièrement du Ministère des transports, chargée de développer et de promouvoir le projet. Les études techniques sont lancées et les premiers objectifs sont fixés par le gouvernement travailliste. Un projet plus détaillé et surtout le tracé de la ligne prévue pour la phase 1 sont publiés en mars 2010, suivant les recommandations des experts. Arrivé au pouvoir en mai 2010, le gouvernement Cameron hérite du dossier, mais ne révise que de façon marginale le projet travailliste. Les consultations et le débat public sont entamés en février 2011 pour une durée de 6 mois. En janvier 2012, le Ministère des transports publie un document final, précisant un certain nombre de points, notamment le calendrier, et corrigeant marginalement le tracé pour répondre à quelques requêtes émanant du débat public. D’autres aménagements ponctuels sont annoncés en avril 2013, notamment la construction de tunnels sous des zones sensibles dans les boroughs périphériques de Londres.

\section{2. ... mais pourtant très contesté}

31 En effet, si les trois principaux partis britanniques (travaillistes, conservateurs et libdem) reconnaissent officiellement la nécessité de construire cette nouvelle ligne, 
certains de leurs élus, des groupes d'intérêts et surtout des associations locales s'opposent à ce projet. Allant d'un rejet général à la contestation d'aspects précis et très localisés, ces réactions ont des motivations très diverses et expriment des représentations très différentes $\mathrm{du}$ territoire, à différentes échelles. Parmi les opposants, on trouve :

- Ceux qui s'opposent au projet, essentiellement à cause de son coût. Ils dénoncent la construction de la nouvelle ligne pour son impact sur les finances publiques et donc sur les impôts. Ils contestent les prévisions de rentabilité du gouvernement ${ }^{10}$, en montrant que la HS2 n'atteindra jamais ses objectifs. Parmi eux, on peut citer certains élus mécontents : on les trouve principalement le long du tracé prévu (voir figure 4). Ainsi, 19 autorités locales se sont regroupées dans le collectif $51 \mathrm{~m}$ : selon eux, la HS2 reviendra à 51 millions de livres sterling - d'où leur nom - pour chacune des circonscriptions parlementaires du pays. On peut néanmoins percevoir chez eux une forme de frustration de voir la ligne à grande vitesse traverser leur territoire, mais sans s'y arrêter ${ }^{11}$.

Ils sont soutenus par certains think-tanks de conseil économique (New Economics Foundation, Centre for Economics and Business Research), des organisations patronales (Institute for Directors, Federation of Small Businesses), des associations de contribuables (Taxpayers Alliance) ou le parti europhobe UK Independence Party (UKIP) qui souligne le coût très élevé d'un projet conçu selon lui pour répondre à des exigences européennes.

- Ceux qui ne contestent pas l'idée d'une HS2, mais qui dénoncent certains aspects généraux du projet. Ils reconnaissent le besoin de construire de nouvelles infrastructures ferroviaires ou le recours à la grande vitesse, mais réclament certains aménagements et plusieurs modifications du projet, notamment sur le tracé. Parmi eux, on trouve beaucoup d'associations de sauvegarde de l'environnement, du patrimoine et des paysages. C'est par exemple le cas de la CPRE, des Wildlife Trusts, de la Royal Society for the Protection of Birds, du National Trust, de la Society for the Protection of Ancient Buildings ou d'ONG comme Friends of the Earth ou Greenpeace. Toutes ces organisations s'inquiètent de l'impact de la ligne sur les espaces traversés, dont certains sites naturels classés et protégés. Elles ont signé une charte commune en juin 2011, la Right Lines Charter, pour persuader le gouvernement de revoir son projet et de prendre davantage de temps pour la concertation.

Le Green party, la principale formation politique écologiste, partage cette position. En effet, il soutient l'idée d'un train capable de concurrencer et de se substituer aux transports routiers et aériens plus polluants. Toutefois, il réclame des trains moins rapides, circulant à 300-320 km/h, comme sur la HS1 ou les LGV françaises, au lieu des $400 \mathrm{~km} / \mathrm{h}$ annoncés. Ainsi, les déplacements seraient moins énergivores et certaines contraintes pour le tracé de la nouvelle ligne disparaitraient. Les écologistes réclament que la HS2 suive les corridors déjà existants des voies ferrées classiques ou des autoroutes, pour réduire l'impact paysager. La position du Green party s'est peu à peu durcie depuis l'arrivée au pouvoir du gouvernement Cameron et la confirmation de ses engagements en janvier 2012, ils réclament désormais que le projet soit gelé pour être totalement repensé.

- Ceux qui s'opposent à la HS2 pour des questions locales. Dès les annonces officielles sur le tracé de la future ligne, de nombreux mouvements locaux se sont constitués pour le dénoncer. Ces associations de riverains sont souvent relayées et soutenues par les élus locaux. Des députés de tous bords politiques contestent 
également le tracé, notamment quand leur circonscription est concernée. Ces riverains contestent la HS2 pour des questions de nuisances visuelles ou sonores, et de protection $\mathrm{du}$ patrimoine, de l'environnement ou des paysages, entrant pleinement dans la mobilisation NIMBY.

Pour peser davantage, ces associations se sont regroupées dans deux collectifs, Stop HS2 et HS2 Action Alliance, eux-mêmes membres de l'Action Groups Against High Speed Two (AGAHST). L'AGAHST, qui se présente comme une fédération regroupant 72 associations locales de lutte contre la HS2, entend proposer et promouvoir des solutions alternatives ${ }^{12}$. Ces associations de riverains contestent ce qu'elles appellent les « 6 mythes de la HS2 » : selon elles, la nouvelle lignene créera pas un mode de transport vert; ne permettra pas de réduire les disparités régionales; ne sera pas rentable ; ne répondra pas à la saturation du transport ferroviaire ; ne concurrencera pas l'avion et n'est pas nécessaire pour développer un réseau à grande vitesse aux normes européennes ${ }^{13}$. Ainsi, individuellement ces multiples petites associations d'opposants ont des préoccupations très locales, mais collectivement elles reprennent des thèmes et des arguments beaucoup plus généraux (voir figure 3).

Bien entendu, cette typologie établie sur les grandes lignes du discours des différents acteurs est très ouverte : les liens entre ces groupes sont très étroits et leurs arguments se croisent souvent. Ils mènent des actions communes, participent aux mêmes manifestations et engagent des recours conjoints auprès des tribunaux.

Figure 3 : Slogans, logos et arguments percutants de Stop HS2

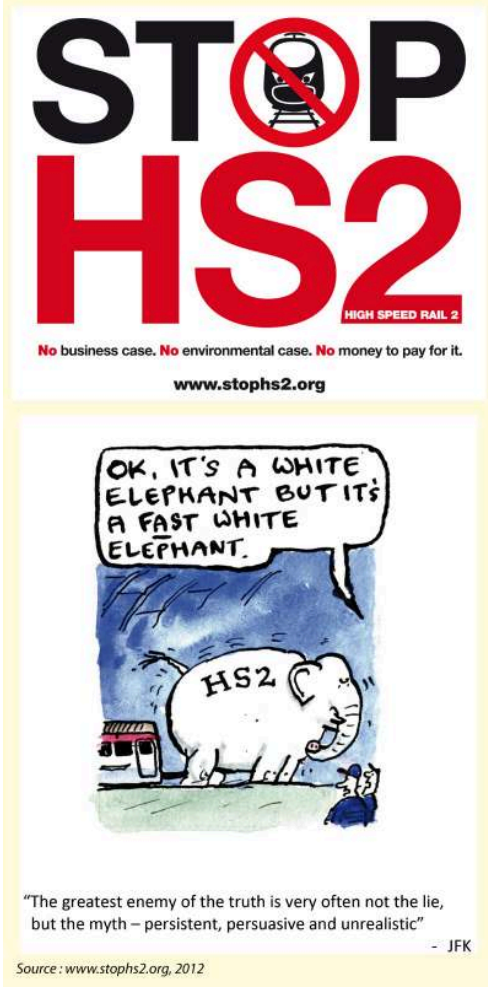

La figure 4 montre que des riverains se sont mobilisés pour défendre des territoires urbains, comme au cœur de Londres, où la construction de la ligne nécessiterait la destruction de logements (boroughs de Camden et de Hillingdon) et menace la tranquillité de certains secteurs résidentiels, comme la très cossue colline de Primrose 
Hill ou le quartier très populaire de Camden. Toutefois, l'essentiel des organisations a été créé dans des espaces ruraux périurbanisés. La carte montre bien la concentration des associations dans la périphérie rurale de Londres, notamment dans les Chilterns, et dans les très chics campagnes du Warwickshire, très proches de Birmingham et Coventry.

Figure 4 : La HS2, un projet contesté particulièrement dans les territoires périurbains

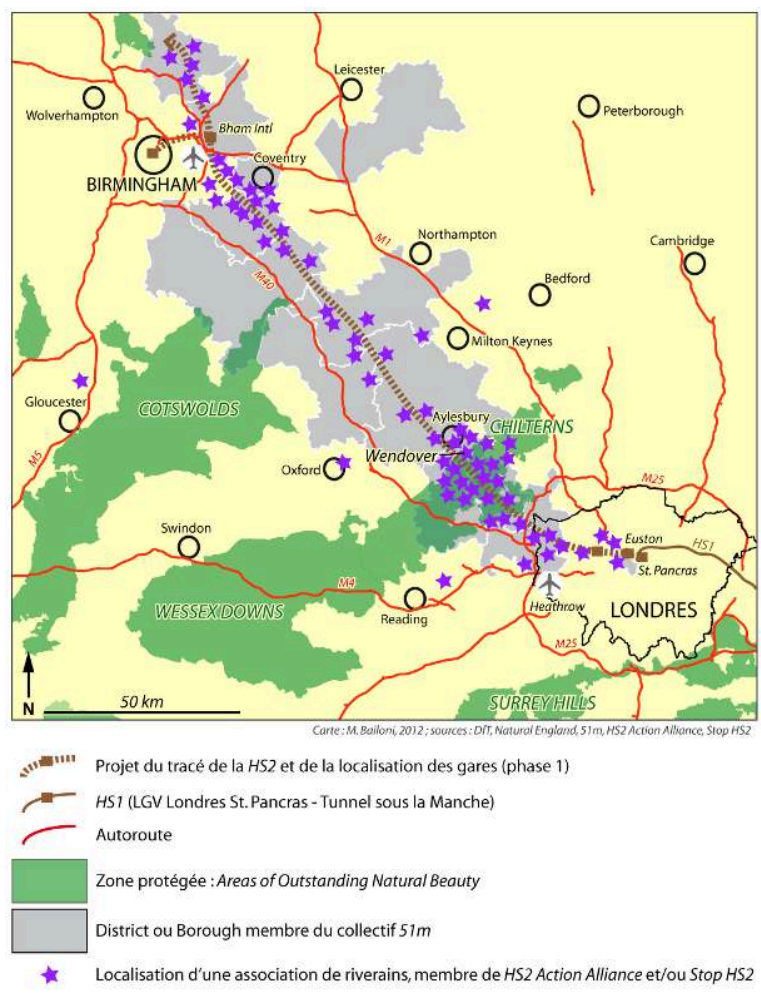

\section{La mobilisation de Wendover, modèle de la contestation NIMBY}

L'exemple de la mobilisation générale du bourg de Wendover illustre parfaitement ces contestations NIMBY. Cette petite ville du Buckinghamshire, à $60 \mathrm{~km}$ du centre de Londres et à $130 \mathrm{~km}$ de Birmingham, compte environ 8.000 habitants. De nombreux résidents travaillent à Londres et font le trajet quotidiennement, puisque Wendover n'est qu'à 45 minutes en train de la gare de Marylebone. Avec un taux de chômage, un revenu moyen, des indicateurs de santé ou un taux de délinquance meilleurs que la moyenne nationale ou même régionale, Wendover apparait comme une ville particulièrement favorisée et prospère. Le bourg est un fief tory: ses élus locaux sont tous conservateurs ${ }^{14}$. La circonscription d'Aylesbury, où se situe Wendover, élit un député conservateur depuis les années 1920, c'est l'un des bastions les plus sûrs du parti.

Les habitants et les élus de Wendover sont particulièrement mobilisés contre le projet de la HS2, car la nouvelle ligne doit passer juste au Sud du bourg, à quelques centaines de mètres du centre, sans bien sûr s'y arrêter (figure 5). Beaucoup de résidents craignent pour leur tranquillité, mais également pour la qualité de leur cadre de vie. Wendover est en effet un bourg rural très caractéristique du centre de l'Angleterre, presqu'un cliché, avec ses petites maisons blanches aux toits de chaumes et ses pubs 
traditionnels. De plus, la petite ville se situe au pied des Chiltern Hills, un ensemble de collines très fréquentées par les randonneurs. Elles constituent une zone protégée sous le statut de area of outstanding natural beauty («espace d'une beauté naturelle remarquable ») depuis 1965. Cette classification doit favoriser en principe la protection des paysages naturels et ruraux considérés comme exceptionnels, par des règles d'aménagements et de pratiques agricoles relativement strictes, sans toutefois relever du statut plus contraignant des parcs naturels ${ }^{15}$. Le périmètre classé s'étend tout autour de Wendover, jusqu'aux limites du bâti. La défense des Chiltern Hills est un thème très fédérateur dans la mobilisation locale contre la HS2. Les symboles de la contestation semblent omniprésents à Wendover (figure 6). Des affiches ont été appliquées sur tous les réverbères du centre, sur les vitrines des magasins et aux fenêtres des maisons pour dénoncer le projet. Des panneaux indicateurs ont été fixés sur les chemins de randonnée des environs pour montrer où passera la ligne et pour suggérer son impact sur la beauté et la tranquillité des lieux. La communauté locale a créé une association, Wendover HS2, qui est membre des différents réseaux d'opposants. Protection du patrimoine et des paysages, défense de la tranquillité ou lutte contre les nuisances sont autant d'arguments très classiques, presque incontournables, des mobilisations NIMBY.

Des élus soutiennent cette mobilisation : bien entendu les conseillers locaux de Wendover, mais également l'ensemble des conseils de district ${ }^{16}$ et de comté, tous deux membres de $51 \mathrm{~m}$. Le député local, David Lidington qui est par ailleurs Secrétaire d'Etat à l'Europe dans le gouvernement Cameron, est également opposé au projet de la HS2, tel qu'il est proposé. Son site internet ${ }^{17}$ semble montrer à ses concitoyens qu'il consacre une grande partie de ses activités à lutter contre le passage de la ligne dans sa circonscription, en interpellant les différents acteurs impliqués ${ }^{18}$.

Figure 5 : Le projet de la HS2 à Wendover, objet d'une vive contestation NIMBY

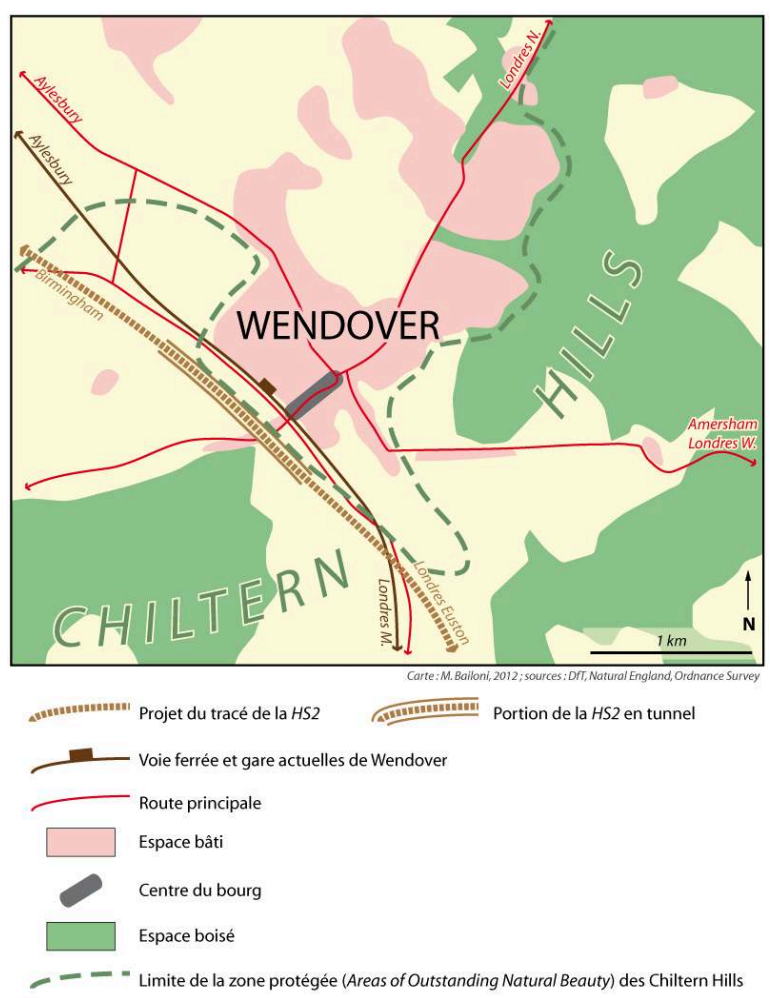


Figure 6 : Mobilisation NIMBY dans le village modèle de Wendover

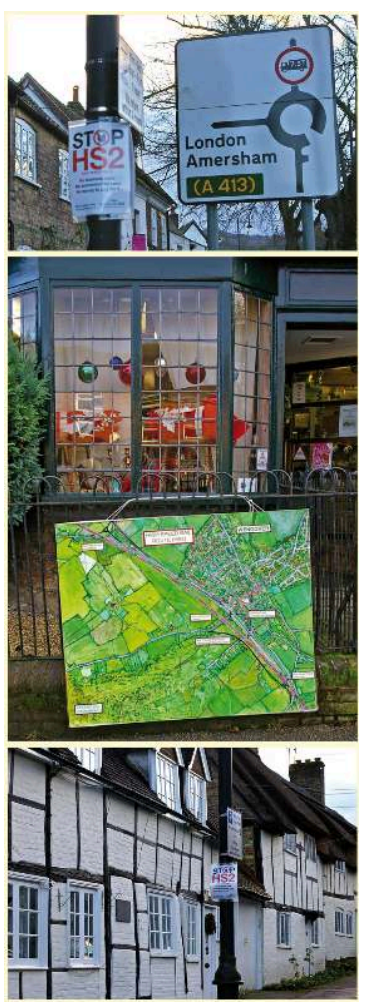

Photos : Bailoni, décembre 2011 quotidiens nationaux et par la $B B C$, parce qu'elle apparait exemplaire à plusieurs titres. D'abord, elle semble impliquer l'ensemble de la ville. La cohésion de cette communauté locale face à l'Etat, à l'administration et aux grands décideurs économiques, cette lutte de David contre Goliath, attire forcément une certaine sympathie. Ensuite, l'esthétique du village-modèle, cliché de l'Angleterre rurale, au milieu d'espaces naturels remarquables, donne au sujet un caractère très photogénique. La mobilisation pour sauvegarder l'environnement, les paysages et la quiétude des Chiltern Hills, un espace très connu des Anglais et très fréquenté le weekend par les Londoniens, suscite une forte solidarité, même loin de Wendover. Enfin, la lutte d'élus locaux conservateurs contre un projet soutenu par un Premier ministre conservateur renforce l'intérêt de ce conflit local et donne un très bon sujet de reportage. Dans ce type de contestation, l'image est en effet très importante, elle conditionne souvent la médiatisation, et donc l'intérêt, la sympathie et la solidarité, même chez les personnes non-concernées directement par le projet en question. D'autres conflits d'aménagement, comme ceux du Larzac, de Plogoff ou de l'hôpital de Carhaix en France, ont déjà montré l'importance de l'image et des médias sur l'efficacité de la mobilisation (Subra, 2007). En France, au Royaume-Uni, comme ailleurs, les mécanismes de la contestation NIMBY sont souvent similaires.

D'ailleurs, la contestation locale et la pression des élus locaux ont abouti sur une révision du projet. En effet, l'une des principales modifications du gouvernement Cameron proposées en janvier 2012, concerne directement Wendover et entend répondre aux inquiétudes des riverains. En effet, il est prévu de construire un tunnel de 1,3 kilomètre au niveau du bourg, pour atténuer les nuisances du train. Le

Revue Géographique de l'Est, vol. 52 / 3-4 | 2012 
gouvernement souligne que les voies seront en très grande partie encaissées à travers les Chiltern Hills pour réduire les impacts sonores et visuels de la HS2. Toutefois, ces quelques aménagements n'ont pas calmé la contestation qui reste vive, tout au long du tracé. En effet, dans les Chiltern Hills, les opposants estiment que rien n'a réellement changé puisque la HS2 coupera l'espace protégé. A Wendover, les résidents estiment que le tunnel est certes un premier pas, mais très insuffisant puisque les nuisances visuelles et sonores subsisteront de part et d'autre.

Cet exemple de contestation NIMBY montre les difficultés d'aménager les espaces périurbains, notamment quand ils présentent un attrait particulier. Dans le discours et les représentations des opposants à la HS2 dans les Chiltern Hills, ce sont les clichés d'une Angleterre idyllique qui sont mobilisés, à travers la défense de paysages ruraux et d'espaces dits « naturels », bien que forgés depuis des siècles par l'Homme.

\section{Des enjeux locaux aux intérêts politiques nationaux, un conflit à plusieurs échelles}

46 La question de la HS2 montre également que les enjeux et les mécanismes du conflit d'aménagement évoluent considérablement selon l'échelle et le niveau d'analyse. En passant de Wendover à l'échelon national, les intérêts et les discours des acteurs impliqués sont souvent très différents, ce qui complexifie encore la question.

La HS2 est en effet un dossier politique très sensible pour le gouvernement Cameron. La ligne traverse une zone de force électorale majeure de son parti, et, comme l'a montré l'exemple de Wendover, la majorité des élus locaux et des députés conservateurs des territoires concernés se sont déclarés opposés au projet. L'exécutif et l'état-major du parti apparaissent également divisés. En effet, face à la révision des prévisions de croissance et surtout face à une mobilisation locale de plus en plus influente, plusieurs ministres seraient même opposés au projet selon la presse, notamment le Chancelier de l'Echiquier George Osborne (The Spectator, 16 juin 2012). La HS2, et au-delà de nombreux projets d'aménagement en milieu rural comme la question des éoliennes ${ }^{19}$, seraient ainsi des sujets de tensions très importantes dans les luttes d'influence internes au parti conservateur (The Guardian, 6 février 2012).

Les résultats des élections locales de mai 2013 renforcent les mécontentements au sein du parti tory. En effet, le UKIP, formation de droite europhobe au discours volontiers populiste, a gagné de nombreux sièges d'élus locaux au cours de ce scrutin, particulièrement dans des zones de force historiques des conservateurs. Ce parti a ainsi remporté six sièges au conseil de comté du Buckinghamshire, bastion tory, où il n'en avait auparavant aucun ${ }^{20}$. Ces résultats expriment le mécontentement grandissant de l'électorat traditionnel de droite face à la politique gouvernementale sur des dossiers divers (Europe, mariage gay, politique économique), dont bien sûr le projet de la HS2. Le UKIP bénéficie ici localement de son opposition à la ligne à grande vitesse. Il a d'ailleurs obtenu ses meilleurs résultats au centre du comté, dans les territoires où devrait passer la HS2 : ainsi, la circonscription dont dépend Wendover a été remportée par le candidat UKIP. Ce vote UKIP n'exprime pas un rejet des élus conservateurs locaux - le conseil du Buckinghamshire s'étant prononcé et militant contre le projet de la HS2 - mais sanctionne la politique du gouvernement Cameron. Les tories sont très inquiets d'une progression encore plus forte de l'UKIP lors des prochaines élections législatives, quand l'enjeu politique apparaitra réellement national. Avec ce dossier, le Premier ministre, se retrouve donc dans une situation très difficile, alors qu'il doit constamment négocier 
entre l'aile droite de son parti et ses partenaires lib-dem au sein de la coalition gouvernementale.

La position du gouvernement est d'autant plus complexe que le parti conservateur avait dénoncé très fermement certains projets proposés par ses prédécesseurs travaillistes, notamment l'extension d'Heathrow, en critiquant le manque de concertation avec les territoires locaux. Les conservateurs aimaient alors se présenter comme les défenseurs des espaces ruraux menacés par la politique des travaillistes. La fracture électorale historique ville/campagne au Royaume-Uni ${ }^{21}$ allait d'ailleurs dans leur sens. Mais l'alternance politique et l'arrivée au pouvoir changent les choses. Les opposants à la HS2 critiquent la position des dirigeants du parti et leur double discours : quand ils étaient dans l'opposition et contestaient certains projets, ils affirmaient défendre "la démocratie locale ", aujourd'hui qu'ils sont au pouvoir et soutiennent certains programmes, ils dénoncent le «nimbysme » des opposants.

La position du parti travailliste a également évolué avec l'alternance politique. Dans l'opposition, le Labour ne peut s'opposer à un projet dont il a conçu l'essentiel des modalités quand il était au pouvoir. Mais il se dit désormais ouvert à d'éventuelles modifications, parfois de fond, pour répondre aux critiques et aux revendications des associations, notamment sur le tracé de la ligne. Les travaillistes demandent ainsi davantage de concertation, critiquant au passage les conservateurs sur leurs pratiques.

51 Finalement, la mobilisation pourrait aboutir, grâce aux divisions internes et aux intérêts politiques du parti conservateur. Ainsi, depuis l'été 2012, la presse britannique relaie régulièrement des rumeurs sur un possible abandon de la HS2. En effet, le gouvernement pourrait finalement revenir sur sa décision et autoriser la construction d'une $3^{\text {ème }}$ piste à Heathrow, convaincu par les lobbys de l'aérien et par des impératifs économiques. D'après ses engagements de 2010, le gouvernement Cameron n'a que suspendu ce dossier, et a promis de ne pas le rouvrir avant 2015, c'est-à-dire après les prochaines élections législatives. Or, les travaux de la première phase de la HS2 ne doivent pas commencer avant 2017. Les conservateurs pourraient ainsi choisir d'étendre Heathrow et de sacrifier la HS2 pour limiter les protestations. Ils ne peuvent en effet se permettre d'affronter deux fronts de contestation majeurs dans leur principale zone de force électorale.

\section{Conclusion}

52 A travers l'exemple du projet de la HS2, on perçoit l'influence considérable de la contestation locale sur les questions d'aménagement, puisqu'elle complique très fortement la décision politique. Les élus, et notamment quand ils ont le pouvoir, sont en effet souvent confrontés à des enjeux politiques contradictoires et à des intérêts différents selon le niveau d'analyse. D'ailleurs, au Royaume-Uni comme en France, les grands projets d'aménagement ne semblent aboutir qu'au terme de très longues années de réflexion, de contestation, de concertation et d'atermoiement. Ainsi, au pays pionnier du transport ferroviaire, il a fallu attendre 13 ans après l'ouverture du Tunnel sous la Manche pour qu'il soit relié à grande vitesse à Londres. Le terminal 5 d'Heathrow a quant à lui ouvert 30 ans après les premières études. La mobilisation des riverains et la contestation dans les espaces périurbains ont en effet considérablement retardé les deux aménagements. Malgré ces expériences, les projets de la $3^{\text {ème }}$ piste d'Heathrow, voire de la HS2, semblent suivre cette même voie. 
53 Malgré des débats publics, des concertations ou des programmes respectueux des préoccupations environnementales, les phénomènes de contestation se généralisent et tendent à systématiquement retarder, voire faire avorter certains projets. Un aménagement du territoire consensuel, qui ne provoquerait pas de conflit, semble ainsi illusoire. Si ces difficultés et ces obstacles concernent les aménageurs et les décideurs politiques de nombreux types de territoires en Europe et ailleurs dans le monde, l'espace périurbain de Londres offre un exemple particulièrement significatif. D'ailleurs, dans ce territoire, le processus de contestation et de blocage est tel que l'on pourrait s'interroger sur la réelle possibilité d'aménager et de transformer l'espace. Finalement, est-ce que cette campagne anglaise, véritable patrimoine national et symbole identitaire, ne tend pas à devenir une forme de grand musée à ciel ouvert, de la même manière que certains centres-villes historiques?

\section{BIBLIOGRAPHIE}

Appert M., Bailoni M., Papin D., 2012, Atlas de Londres, Paris, Autrement, 96 p.

Bailoni M., 2007, La question régionale en Angleterre : nouvelles approches politiques du territoire anglais, thèse, Institut Français de Géopolitique - Université Paris 8, 560 p.

Bailoni M., 2009, « L'aménagement du territoire en Angleterre : New Labour, new planning ? », in Jean Y., Baudelle G., L'Europe, aménager les territoires, Paris, A. Colin, p.183-197.

Bailoni M., 2010, « Le Nord de l'Angleterre, l'affirmation d'un territoire politique et identitaire »,Hérodote, n¹37, Paris, La Découverte, p.70-92.

Bailoni M, Edelblutte S., Tchékémian A., 2012, « Agricultural landscapes, heritage and identity in peri-urban areas in Western Europe ", European Countryside, Vol.4, n², p. 147-161.

Baker A., 2010, « Controlling the growth of Cambridge: challenges to planning its built environment », Annales de Géographie, n673, p.293-304.

Barclay C., 2012, Green Belt, standard note $n^{\circ} 934$, Londres, House of Commons Library, 8 p.

Barker K., 2006, Barker review of land use planning - Final report / recommendations, Londres, HM Treasury, $226 \mathrm{p}$.

Bunce M., 1994, The Countryside Ideal, Londres, Routledge, 232 p.

Champion T., 2001, « The continuing urban-rural population movement in Britain: trends, patterns, significance ", Espace, populations, sociétés, vol. 19, n¹/2, p.37-51.

Champion T., Fisher T., 2004, « Migration residential preferences and the changing environment of cities ", in Boddy M., Parkinson M., City matters: competitiveness, cohesion and urban governance, Bristol, Policy Press, p. 111-128.

Charlier B., 2005, « Caractéristiques et évolution des conflits d'usage dans les espaces ruraux et périurbains ", in Guérin M., Conflits d'usage à l'horizon 2020. Quels nouveaux rôles pour l'Etat dans les espaces ruraux et périurbains?, Paris, La Documentation Française, p. 17-46. 
Charlier B., 1999, La défense de l'environnement : entre espace et territoire, thèse, Université de Pau et des Pays de l'Adour, 753 p.

CPRE, Natural England, 2010, Green Belts : a greener future, $138 \mathrm{p}$.

Cullingworth B., Nadin V., 2006, Town and country planning in the UK, Londres, Routledge, 587 p. Department for Communities and Local Governement (DCLG), 2012, National Planning Policy Framework, 65 p.

Department for Transport (DfT), 2009, Britain's transport infrastructure: High speed two, 25 p. Department for Transport (DfT), 2012, High speed rail : investing in Britain's future - Decisions and next steps, $120 \mathrm{p}$.

Dziedzicki J-M., 2001, Gestion des conflits d'aménagement de l'espace : quelle place pour les processus de médiation?, thèse, Université de Tours / CESA, 443 p.

Gilbert Y., 2010, « Migrations urbaines en milieu rural : diversification sociale et recomposition du politique », Espaces et sociétés, n¹43, 3/2010, p. 135-149.

Gilg A., 2005, Planning in Britain: Understanding and Evaluating the Post-War System, Londres, Sage, $224 \mathrm{p}$.

Guérin M., 2005, Conflits d'usage à l'horizon 2020 - Quels nouveaux rôles pour l'Etat dans les espaces ruraux et périurbains?, Paris, La Documentation française, 205 p.

Hall P., 2002, Urban and regional planning, Londres, Routledge, 237 p.

Hall P., Thomas R., Gracey H., Drewett R., 1973, The Containment of Urban England, Londres, Allen and Unwin, $393 \mathrm{p}$.

Howkins A., 2001, «Rurality and English History », in Morley D., Robins K., 2001, British Cultural Studies, Oxford, Oxford University Press, p.145-156.

Howkins A., 2007, « What is the countryside for? Agriculture, leisure and the English countryside 1900-2000 », Revue Française de Civilisation Britannique, vol. XIV, n², p.165-180.

Le Caro Y., 2002, Usages récréatifs de l'espace agricole, thèse, Université de Rennes 2 - HauteBretagne, $779 \mathrm{p}$.

Lecourt A., 2003, Les conflits d'aménagement : analyse théorique et pratique à partir du cas breton, thèse, Université de Rennes 2 - Haute Bretagne.

Lecourt A., Faburel G., 2005, « Comprendre la place des territoires dans les conflits d'aménagement et d'environnement. Une application aux espaces ruraux », conférence Faire Campagne. Pratiques et projets des espaces ruraux aujourd'hui, Université de Rennes 2.

Mathis C-F., 2010, In Nature We Trust - Les paysages anglais à l'ère industrielle, Paris, Presses de l'Université Paris-Sorbonne, 685 p.

Matless D., 1998, Landscape and Englishness, Londres, Reaktion Books, 357 p.

Murdoch J., 1997, Why do people move to the countryside? A report for the Countryside Commission, Cardiff, Department of City \& Regional Planning, University of Wales.

Murdoch J., Lowe P., 2003, « The preservationist paradox: modernism, environmentalism and the politics of spatial division », Transactions of the Institute of British Geographers, ${ }^{\circ} 28$, p.318-332.

OCDE, 2011, Economic surveys - United Kingdom overview, 18 p. 
Office of the Deputy Prime Minister (ODPM), 1995 (révisé en 2001), Planning Policy Guidance 2: Green Belts, 25 p.

Phillips M., 1993, « Rural gentrification and the process of class colonisation », Journal of Rural Studies, vol.9, $n^{\circ} 2$, p. 123-140.

Pistre P., 2010, « Mobilisation dans les campagnes toulousaines : conflit d'aménagement et tensions locales », Espaces et sociétés, ${ }^{\circ} 143,3 / 2010$, p. 151-169.

Poulot M., 2008, « Les territoires périurbains : « fin de partie » pour la géographie rurale ou nouvelles perspectives?", Géocarrefour, vol.83, n4.

Richard F., 2009, « La gentrification des « espaces naturels » en Angleterre : après le front écologique, l'occupation? ", L'Espace politique, n9, 2009/3.

Salomon Carvin J., (2006), « La ville au secours de la campagne, une politique urbaine pour protéger l'Angleterre rurale », Espace et société, n¹26, p. 141-158.

Spiers S., 2009, 2026 A vision for the countryside - Towards our vision, Londres, CPRE, 23 p.

Subra P., 2007, Géopolitique de l'aménagement du territoire, Paris, A. Colin, 326 p.

Tomasin L., 2001, « Un concept pour le décrire : l'espace rural rurbanisé », Ruralia, n9, http:// ruralia.revues.org $/ 250$

Torre A., Lefranc C., 2006, « Les conflits dans les zones rurales et périurbaines. Premières analyses de la presse quotidienne régionale », Espaces et Sociétés, n²124-125, p. 93-110.

Vanier M., 2001, « Le tiers espace, acte II de la périurbanisation », Pouvoirs Locaux, Vol.48, n¹, p. 59-63.

Von der Dunk A., Grêt-Regamey A., Dalang T., Hersperger A., 2011, « Defining a typology of periurban land-use conflicts - A case study from Switzerland », Landscape and Urban Planning, ${ }^{\circ} 101$, p. 149-156.

\section{NOTES}

1. D'autres disciplines s'intéressent également à ce type de conflit, comme le droit, la science politique ou la sociologie (cf. Claeys-Mekdade, 2000, Les conflits d'aménagement. Rapports à la " nature " et rapports sociaux. La Camargue, un cas révélateur, thèse de Sociologie, Université de Provence).

2. Le débat sur la définition de la nation anglaise et sur la distinction entre identité anglaise et identité britannique a été particulièrement nourri depuis les années 1990, en parallèle à des processus politiques particuliers, comme la construction européenne, l'autonomie écossaise ou la remise en cause du multiculturalisme.

3. Comme le souligne C-F. Mathis (2010), l'évolution du terme landscape est très révélatrice : à l'origine il signifiait une peinture représentant un paysage, avant de désigner ce paysage luimême.

4. Attention cependant, le seuil officiel de population agglomérée qui distingue ensemble rural et ensemble urbain, est de 10.000 habitants au Royaume-Uni, largement au dessus des 2.000 habitants en France. La population rurale officielle britannique est donc largement surévaluée par rapport à la population rurale française.

5. A. Howkins (2007) rappelle toutefois que l'apparition du camping et l'accès aux chemins de randonnées à travers les propriétés privées, liés au développement des activités de loisirs des 
classes populaires, ont provoqué de nombreux conflits locaux dans toutes les campagnes anglaises pendant l'entre-deux-guerres.

6. Cette expression désigne les comtés traditionnels les plus proches de Londres, formant une ceinture périurbaine sous l'influence directe de la capitale.

7. Ce document, publié en mars 2012 après plus de deux ans de travaux et de consultations, entend synthétiser et rationaliser une série de décrets, de recommandations et de programmes divers, pour mieux définir les grandes orientations, les enjeux et les pratiques de l'aménagement au Royaume-Uni.

8. Ainsi, les mobilisations contre les projets d'aéroport à Notre-Dame-des-Landes, près de Nantes (Lecourt, Faburel, 2005), ou du second aéroport toulousain (Pistre, 2010), emploient un discours et des arguments très proches de ceux des opposants à l'extension d'Heathrow, et expriment une vision quasiment similaire du territoire et de son développement.

9. La HS2 doit arriver à la gare de Londres Euston, alors que l'Eurostar arrive à Saint-Pancras. Les deux gares ne sont distantes que de quelques centaines de mètres. L'interconnexion devrait se faire en amont, empruntant un tout nouveau tunnel et des lignes existantes. Des trains pourraient également arriver directement à Heathrow pour éviter aux passagers des Midlands ou du Nord un changement dans Londres. Une gare est également prévue sur la HS2 pour desservir l'aéroport de Birmingham International.

10. Le gouvernement a d'ailleurs réduit ses prévisions. Au début du projet, la liaison LondresBirmingham devait rapporter 2,4 livres sterling pour chaque livre investie. En avril 2012, le ratio est révisé à 1,2 livre de bénéfice pour une livre investie (The Guardian, 11 avril 2012).

11. Ainsi, dans ce collectif, on retrouve le Conseil du borough londonien de Hillingdon, dont le territoire sera seulement traversé par la ligne. Au contraire, le borough de Hammersmith et Fulham soutient fermement le projet. En effet, il est prévu d'y construire la gare d'old Oak Common, où se fera l'interconnexion entre la HS2, Crossrail (le futur RER londonien), le Heathrow Express, les grandes lignes vers l'Ouest et le Sud-Ouest de Londres, et la future branche vers la HS1. Le Conseil d'Hammersmith et Fulham souligne l'intérêt pour le renouveau urbain local d'une telle gare.

12. Selon le site internet de l'AGAHST : www.betterthanhs2.org

13. On retrouve les démonstrations contestant ces « 6 mythes » sur les sites internet des deux collectifs, www.hs2actionalliance.org et www.stophs2.org. Ces arguments sont régulièrement repris par tous les autres sites ou blogs contestant le projet.

14. Administrativement, Wendover constitue une civil parish ("paroisse civile»), échelon plus traditionnel et symbolique que véritablement politique, dont les compétences sont limitées à des actions culturelles locales. Elle fait partie du district d'Aylesbury Vale, premier véritable échelon politique local, équivalent aux communes. Le district fait partie du comté du Buckinghamshire, second échelon politique local. Wendover est représentée par trois élus au conseil du district, et par un élu au conseil du comté. Ces quatre élus sont tous conservateurs. Aux dernières élections du comté (2009), le candidat tory a recueilli $54,7 \%$ des voix, loin devant le lib-dem $(26,4 \%)$ ou le travailliste (5\%). Aux dernières élections du district, le Labour n'a même pas présenté de candidat à Wendover.

15. Les areas of outstanding natural beauty (AONB) ont été créées par la loi National parks and access to the countryside Act de 1949, qui instaurait également les parcs naturels. Ce dispositif s'inscrit dans les grandes décisions d'après-guerre pour limiter l'étalement urbain, protéger les franges rurales et créer de véritables outils d'aménagement périurbain. Il existe 40 AONB en Angleterre et au pays de Galles, et 9 en Irlande du Nord.

16. Le site internet du district d'Aylesbury Vale présente même un onglet spécifiquement dédié à la question de la HS2 et de la mobilisation des élus contre le projet et le tracé : http:// www.aylesburyvaledc.gov.uk/community-living/high-speed-rail-link/ (consulté en mai 2013). 
17. http://davidlidington.wordpress.com/ (consulté en mai 2013).

18. Il est parfois complexe de distinguer le véritable engagement personnel et la stratégie électoraliste dans le positionnement des élus locaux dans ce type de conflit. Des auteurs anglosaxons ont même inventé l'acronyme NIMTO (Not In My Term of Office), « pas au cours de mon mandat », pour évoquer le comportement d'élus soucieux de satisfaire leur électorat.

19. L'énergie éolienne terrestre est en effet un sujet de division interne à la coalition au pouvoir. Au début de l'année 2012, 101 députés tory - plus deux travaillistes et trois lib-dem - ont écrit au Premier ministre pour lui demander de supprimer les aides publiques au secteur de l'éolien terrestre (The Sunday Telegraph, 5 février 2012). Ces députés, en très grande majorité issus de circonscriptions rurales ou périurbaines, dénoncent le gaspillage de l'argent des contribuables pour soutenir une production d'énergie « inefficace et intermittente». Mais ils entendent surtout défendre les paysages. Ces députés s'inscrivent dans une logique NIMBY, s'engageant pour empêcher l'implantation de nouveaux parcs éoliens dans leur circonscription (The Guardian, 6 février 2012). Le Premier ministre - qui avait déclaré que son gouvernement était « le plus vert » de l'histoire - est dans une situation délicate, pris en tenaille entre ses députés, ses engagements en faveur des énergies renouvelables et son vice-Premier ministre, le lib-dem Nick Clegg, soutenant fermement l'énergie éolienne.

20. Suite aux élections de mai 2013, les conservateurs conservent néanmoins la majorité au conseil du comté de Buckinghamshire, avec 36 sièges (sur 49), devant l'UKIP, les lib-dems (5 sièges) et les travaillistes (1 siège). Le dernier siège du conseil a été remporté par un candidat indépendant. Mais le parti UKIP est passé de 13,5\% des voix en 2009 à $27 \%$ en 2013, alors que les partis de la coalition au pouvoir, les conservateurs $(49,2 \%$ en 2009 ; 41\% en 2013) et les lib-dems $(28,1 \% ; 14,9 \%)$, ont nettement reculé.

21. La fracture électorale entre des espaces ruraux acquis très largement aux conservateurs et des territoires urbains votant très majoritairement travailliste est très nette en Angleterre, au Nord comme au Sud du pays, et se vérifie à chaque scrutin, même si certaines villes comme Londres basculent parfois. Cette fracture politique peut apparaitre comme une forme de clivage identitaire dans la société anglaise (Bailoni, 2007).

\section{RÉSUMÉS}

Cet article analyse les conflits d'aménagement et le phénomène NIMBY (« Not In My Back Yard») dans les espaces ruraux périphériques de Londres. Ce territoire, l'un des plus riches du pays, est confronté au processus de périurbanisation et d'étalement urbain. Cet espace idéalisé et symbolique de la campagne anglaise est au cœur de nombreux enjeux politiques, électoraux, économiques et environnementaux. Toutefois, malgré des débats publics, des concertations ou des programmes respectueux des préoccupations environnementales, les phénomènes de contestation se généralisent et tendent à systématiquement retarder, voire faire avorter certains projets. Un aménagement du territoire consensuel, qui ne provoquerait pas de conflit, semble ainsi illusoire. Pour étudier ces enjeux, les mécanismes des conflits d'aménagement et les perceptions des différents acteurs impliqués, cet article développe plus précisément le projet de ligne à grande vitesse entre Londres et Birmingham.

This paper analyzes the development of planning conflicts and the NIMBY (« Not In My Back Yard ») phenomenon in rural surroundings of London. This area, one of the richest of the 
country, is confronted with the process of suburbanization and urban sprawl. This idealised and symbolic space of the English countryside is at the heart of many political, election, economic and environmental issues. However, despite public debates, consultations and environmentally friendly plans, the process of protest tends to be widespread, to systematically delay projects or even to derail them. In this context, a consensusal planning project that would not cause conflict, seems illusory. To study these issues, the process of planning conflicts and the perceptions of various stakeholders, this paper develops specifically the HS2 draft, the high-speed rail link between London and Birmingham.

\section{INDEX}

Keywords : Chiltern Hills, conflict, English countryside, HS2, London, national identity, NIMBY, planning, United-Kingdom, urban sprawl

Mots-clés : campagne anglaise, Chiltern Hills, conflit d'aménagement, HS2, identité nationale, Londres, NIMBY, périurbanisation, Royaume-Uni

\section{AUTEUR}

\section{MARK BAILONI}

Géographe, maître de conférences - LOTERR - Université de Lorraine - mark.bailoni@univlorraine.fr 